\title{
Analysis and Simulation of MRC Diversity Reception in Correlated Composite Nakagami-Lognormal Fading Channels
}

\author{
Dinh Thong Nguyen ${ }^{1}$, Quoc Tuan Nguyen ${ }^{2}$, Sinh Cong Lam ${ }^{1}$ \\ ${ }^{1}$ University of Technology, Sydney, Australia \\ ${ }^{2}$ VNU University of Engineering and Technology, Cau Giay, Hanoi, Vietnam
}

Correspondence: Quoc Tuan Nguyen, tuannq@vnu.edu.vn

Manuscript communication: received 7 May 2014, accepted 23 October 2014

\begin{abstract}
The physical meaning of the composite Nakagami-lognormal fading model is not well understood by many researchers using the model. The signal power transfer and transform at the interface between the global lognormal shadowing sub-channels and the local Nakagami multipath sub-channels in the presence of correlation between these diversity sub-channels is rather complex. This is the main reason why a thorough analysis or a simulation model is absent to date for the case of correlated composite Nakagami-lognormal diversity channels. This paper presents a novel technique for the estimation of the probability density function (PDF) of the signal-to-noise (SNR) at the output of a maximum ratio combining (MRC) receiver operating in correlated composite diversity fading channels. The PDF is estimated using the recently proposed two-point lossless moment generating function (MGF) matching technique and a closed-form expression for the bit-error rate (BER) for QPSK signal is consequently presented using the Gauss-Hermite polynomial approximation. The paper also presents the complex Monte-Carlo simulation model for the MRC reception and BER counting in correlated composite Nakagami-lognormal fading channels.
\end{abstract}

Keywords- Nakagami-lognormal, maximum ratio combining (MRC), two point matching, moment generating function (MGF).

\section{INTRODUCTION}

It has long been established that diversity reception is a very effective technique for combating the detrimental effects of channel fading in wireless propagation, and for coherent reception, maximum ratio combining (MRC) is known to be the optimal linear combining technique. The principle of MRC is to sum up the powers, hence the signal-to-noise ratios (SNRs), of the diversity receiving signals. In a general radio propagation scenario over a large urban area, the signal in each diversity path encounters a series/cascade of scattering objects. This leads to what is commonly known as shadow fading referring to the fluctuation in the local mean of the received power as the mobile receiver moves through the shadow of an obstruction object.

The multiplicative nature of the cascading obstructions is widely accepted as having a lognormal distribution and the sum of lognormal powers underlying the analysis of MRC diversity reception has therefore become a challenging topic for research, particularly when the shadowing effects in the diversity channels are correlated. An exact closed-form expression for the probability density function (PDF) of the sum of lognormal RVs is not available to date but a number of analytical approximations do exist.

Most approximation techniques assume that the sum of lognormal RVs can be modelled as another single lognormal RV whose parameters are found by matching of the moments as in the Fenton-Wilkinson (F-W) method [1] or by matching of the log-moments as in the Schwartz-Yeh (S-Y) technique [2] or by matching of the moment generating functions (MGF) at two points as in [3]. A relatively more recent paper in [4] proposes a two-step algorithm, via the Pearson-Type IV distribution, for approximating the lognormal sum and the linear combination with arbitrary weights of the lognormal RVs and shows that the proposed technique is superior to many other techniques currently available. The authors in [3] point out that the popular F$\mathrm{W}$ approximation method in [1] puts more weight on larger SNRs resulting in a poor approximation in the tail portion of the PDF and a better tracking of the head portion.

On the other hand, the S-Y approximation method in [2] suffers from severe error in the head portion but achieves better tracking in the tail portion of the PDF. In [3] by adjusting the position of the two MGF matching points, the PDF tracking error can be controlled and distributed according to applications. In [5] a novel I two-point MGF matching technique is introduced to accurately locate the optimal MGF matching points.

However, in more realistic scenarios in mobile radio in urban areas, the multipath effect at the mobile receiver due to scattering from local scatterers such as buildings in the neighbourhood of the receiver causes a fast fading while the variation in the terrain configuration between the base-station and the mobile receiver causes a slow shadowing. Therefore, the mobile radio signal envelope is usually composed of a small scale 
multipath fading component superimposed on a much larger scale or slower shadowing component [6]. It is well known that the signal envelope of the multipath component can be modelled as a Nakagami- $m$ distributed random variable (RV) [7] or equivalently, its power can be modelled as a gamma distributed RV. Thus the path power gain, hence the SNR, has a mixed gammalognormal distribution which was first proposed in [8] as a generalization of the Rayleigh-lognormal, also known as the Suzuki fading distribution model in [9].

The sum-of-products model in Figure 1 is useful in studying this statistical mix in most physical mobile radio channels. The problem of approximating the power sum of correlated composite gamma-lognormal random variables is again more challenging than that of the power sum of correlated lognormal RVs. While [10] only presented the sum of the independent NakagamiLognormal fading channel, the aim of this paper is to present closed-form expressions for the approximated bit-error rate (BER) of the output from an MRC diversity receiver under the correlated composite Nakagamilognormal fading channels. This is the main contribution of this paper.

The rest of the paper is organized as follows. Section 2 covers Composite Nakagami-lognormal fading channel model. The MRC diversity reception in correlated composite Nakagami-lognormal fading channels algorithm is described in Section 3. Monte Carlo simulation of diversity MRC repetition in correlated composite Nakagami-lognormal fading channels is described in Section 4. Finally, Section 5 gives the conclusions.

\section{Composite Nakagami-Lognormal Fading Channel Model}

It is well known that, if $h$ is the amplitude channel gain and $s(t)$ and $n(t)$ are transmit signal and channel noise then the receive signal can be defined

$$
r(t)=h s(t)+n(t) .
$$

The amplitude channel gain assumed to be constant over the transmission time of a symbol block. $n(t)$ is modeled as a zero-mean additive white Gaussian noise (AWGN) process with one-sided power spectral density $N_{0}$. The received SNR is then

$$
\gamma=\frac{|h|^{2} E_{s}}{N_{0}}
$$

where the signal energy is $E_{s}=E\left[s^{2}(t)\right]$.

In this paper we use the term power gain, $p=h^{2}$, and signal-to-noise ratio, $\gamma$, interchangeably where it is appropriate. If the energy is that of one bit, then we denote $\gamma_{b}$ as the SNR per bit of transmitted information, then we express

$$
\gamma_{b}(p)=p E_{b} / N_{0}
$$

To avoid dealing with distance dependency, we normalize the average channel power gain $E\left|h^{2}\right|=1$, thus

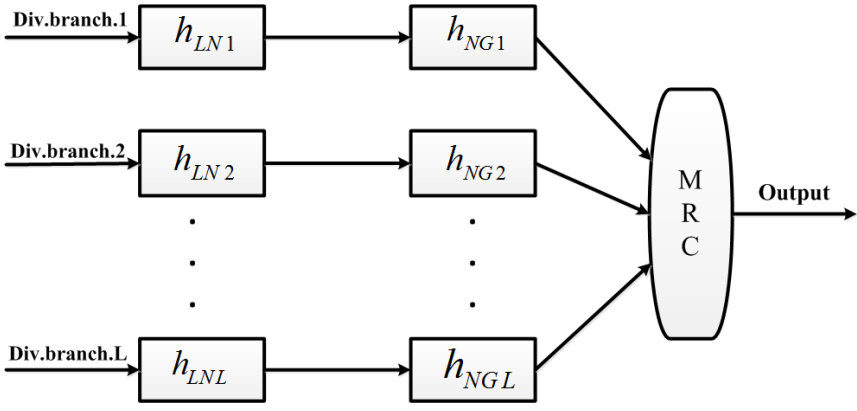

Figure 1. Modeling of diversity branches in a composite Nakagamilognormal fading environment.

making the average received SNR per bit per channel $\bar{\gamma}_{b}=E_{b} / N_{0}$

At high SNR, the symbol-error-rate for rectangular M-QAM in AWGN with $M=2^{k}$ when $k$ is even, is approximated as [9].

$$
\mathrm{SER}_{\mathrm{AWGN}, \mathrm{MQAM}} \approx 4\left(1-\sqrt{\frac{1}{M}}\right) Q\left(\sqrt{\frac{3}{M-1} \gamma_{s}}\right),
$$

in which $\gamma_{s}$ is the average SNR per symbol per channel (without combining), and for equiprobable orthogonal signals the corresponding bit-error-rate is [11].

$$
\mathrm{BER}_{\mathrm{AWGN}, \mathrm{MQAM}}=\frac{M}{2(M-1)} \mathrm{SER}_{\mathrm{AWGN}, \mathrm{MQAM}} \text {. }
$$

For QPSK signaling, $M=4$ and without using Gray code, we have

$$
\operatorname{BER}_{\mathrm{AWGN}, \mathrm{QPSK}}\left(\gamma_{b}\right)=\frac{4}{3} Q\left(\sqrt{2 \gamma_{b}}\right) .
$$

From the model in Figure 1 for mobile radio propagation in urban areas, the path fading from the transmitter to the cluster of buildings in the neighbourhood of the receiver is predominantly large-scale or slow fading with lognormal distribution after the main wave encounters multiplicative reflections and or refractions by obstructions. In most situations the mobile receiver is surrounded by a local cluster of scatterers such as buildings in urban areas, the arriving main wave is scattered into multiple local paths and combined at the receiver causing multipath fading. These local paths arrive at the receiver roughly with the same strength but with different phases, giving rise to small-scale or fast fading with Nakagami- $m$ distributed envelope, i.e. fading power gain is gamma-distributed.

The probability density function (PDF) of the SNR in the composite Nakagami-lognormal fading channel may be derived by equating the local average power of the much faster Nakagami- $m$ fading signal, to the instantaneous power of the much slower arriving lognormal signal. This implies first, a complete transfer of signal power from the arriving global lognormal signal to the local multipath sub-channel, and second, no loss of power in the local multipath channel, i.e. the average power gain of the local Nakagami- $m$ channel can be assumed to be unity. In the diversity composite fading model in Figure 1, in each branch the received channel power gain is the product of the main shadowing lognormal power gain $P_{\mathrm{LN}}=\left|h_{\mathrm{LN}}\right|^{2}$ and 
its local multipath Nakagami- $m$ power gain (gamma distributed) $p_{\mathrm{NG}}=\left|h_{\mathrm{NG}}\right|^{2}$. Since in this paper we are mainly interested in the gamma-distributed power of the local multipath Nakagami- $m$ fading channels, we will denote this as $p_{\mathrm{GM}}$ instead of $p_{\mathrm{NG}}$ in what follows, i.e.

$$
p_{\mathrm{GM}-\mathrm{LN}}(i)=p_{\mathrm{GM}}(i) p_{\mathrm{LN}}(i), \quad i=1,2, \ldots, L
$$

and the average power gain of the local Nakagami- $m$ channel is

$$
\bar{p}_{\mathrm{GM}}=1, \text { for all } i \text {. }
$$

The PDF of the power gain of the Nakagami $(m, \Omega)$ channel, i.e. a gamma $(m, \theta)$ random variable (RV), is

$$
f_{\mathrm{GM}}(G M)=\frac{1}{\Gamma(m)}\left(\frac{1}{\theta}\right)^{m} p_{\mathrm{GM}}^{m-1} \exp \left(-\frac{p_{\mathrm{GM}}}{\theta}\right), \quad m \geqslant 0.5 \text { (9) }
$$

where

$$
\begin{aligned}
\theta & =\frac{\Omega}{m}, \\
\Omega & =E\left[p_{\mathrm{GM}}\right], \\
m & =\frac{\left(E\left[p_{\mathrm{GM}}\right]\right)^{2}}{\operatorname{Var}\left[p_{\mathrm{GM}}\right]},
\end{aligned}
$$

and the PDF of the power gain of the lognormal channel is given by

$$
f_{\mathrm{LN}}\left(P_{\mathrm{LN}}\right)=\frac{1}{p_{\mathrm{LN}}} \frac{\zeta}{\sigma_{z} \sqrt{2 \pi}} \exp \left(-\frac{\left(10 \log _{10} x-\mu_{z}\right)^{2}}{2 \sigma_{z}^{2}}\right),
$$

where the lognormal power fading is modeled as $p_{\mathrm{LN}}=$ $10^{0.1 Z}=\exp (z / \zeta)$ with $Z \sim \aleph\left(\mu_{z}, \sigma_{z}^{2}\right)$ in $\mathrm{dB}$ unit and $\zeta=10 / \log 10$ is the conversion constant between $d B$ and net, and $p_{\mathrm{LN}}$ is in linear unit.

The PDF for the product power gain $p_{\mathrm{GM}-\mathrm{LN}}$ in (7) of each composite Nakagami-lognormal diversity channel, using the Jacobian transformation technique, can be found from (9) and (11) to be

$$
\begin{aligned}
& f_{\mathrm{NG}-\mathrm{LN}}(p)=\int_{0}^{\infty} \frac{1}{\Gamma(m)}\left(\frac{m}{x}\right)^{m} p^{m-1} \exp \left(-\frac{m p}{x}\right) \\
& \frac{1}{x} \frac{\zeta}{\sigma_{z} \sqrt{2 \pi}} \exp \left(-\frac{\left(10 \log _{10} x-\mu_{z}\right)^{2}}{2 \sigma_{z}^{2}}\right) d x, \quad m \geqslant 0.5 .
\end{aligned}
$$

The composite PDF in (12) was first introduced in [7]. If $m=1$, it reduces to the composite Rayleighlognormal, also known as Suzuki distribution [8].

\section{MRC Diversity Reception in CORRELATED Composite NaKagami-lognormal FAdING Channels}

\subsection{MGF of the Sum of Correlated Composite Gamma-lognormal Powers}

In this section, the main contribution of our paper is presented. The mathematical derivation requires the following definitions and notations:

$$
p=\sum_{i=1}^{L} p_{\mathrm{GM}-\mathrm{LN}}(i)=\sum_{i=1}^{L} p_{i}
$$

is the resultant power gain at the output of the MRC combiner. $f_{\mathrm{GM}-\mathrm{LN}}\left(p_{1}, p_{2}, \ldots, p_{L}\right)$ is the joint distribution of the correlated composite gamma-lognormal input powers, and $f_{\mathrm{LN}}\left(x_{1}, x_{2}, \ldots, x_{L}\right)$ is the joint distribution of the lognormal fading component with its associated generating Gaussian vector $\mathbf{z}=\left(z_{1}, z_{2}, \ldots, z_{L}\right)$ in which the elements $z_{i}$ are identically distributed with mean $\mu_{z}$ and variance $\sigma_{z}^{2}$ and are correlated with covariance matrix $C_{z}$, i.e.

$$
f_{Z}(\mathbf{z})=\frac{1}{(2 \pi)^{L / 2}\left|C_{z}\right|^{1 / 2}} \exp \left(-\frac{1}{2}\left(\mathbf{z}-\mu_{z}\right)^{T} C_{z}^{-1}\left(\mathbf{z}-\mu_{z}\right)\right) .
$$

Then the MGF of $p$ is

$$
\begin{aligned}
& M_{p, \operatorname{MRC}}(s)= \\
& \quad \int_{0}^{\infty} \ldots \int_{0}^{\infty} \prod_{i=1}^{L} \exp \left(-s p_{i}\right) f_{\mathrm{GM}-\mathrm{LN}}\left(p_{1}, \ldots, p_{L}\right) d p_{1} \ldots d p_{L}
\end{aligned}
$$

where $s$ is the transform variable in the Laplace domain.

We can immediately recognize that the integral with respect to $p_{1}$ inside the square brackets gives the MGF of the gamma $\left(m, \theta_{i}\right)$-distributed RV [12] with $\theta_{i}=x_{i} / m$, or by directly calculating the integral using [13], we have

$$
\begin{aligned}
& M_{p, \mathrm{MRC}}(s)=\int_{P_{L}}^{\infty} \ldots \int_{p_{2}}^{\infty} \\
& \quad\left\{\int_{0}^{\infty}\left(\frac{1}{1+s x_{1} / m}\right)^{m} f_{\mathrm{LN}}\left(x_{1}, p_{2}, \ldots, p_{L}\right) d x_{1}\right\} d p_{2} \ldots d p_{L} .
\end{aligned}
$$

We proceed in the same way for $p_{2}, p_{3}, \ldots, p_{L}$

$$
\begin{aligned}
M_{p, \operatorname{MRC}}(s) & = \\
& \int_{x_{L}}^{\infty} \ldots \int_{x_{1}}^{\infty} \prod_{i=1}^{L} M_{\mathrm{GM}}\left(s, \frac{x_{i}}{m}\right) f_{\mathrm{LN}}\left(x_{1}, \ldots, x_{L}\right) d x_{1} \ldots d x_{L}
\end{aligned}
$$

where

$$
M_{\mathrm{GM}}\left(s, \frac{x_{i}}{m}\right)=\left(\frac{1}{1+s \frac{x_{i}}{m}}\right)^{m}
$$

is the MGF of the gamma $\left(m, \theta_{i}\right)$-distributed RV [12] with $\theta_{i}=x_{i} / m$ and $x_{i}$ is the local power of the corresponding lognormal diversity branch.

The expression in (14) is rather expected because, in the microdiversity model in Figure 1, the correlation is initially between the global lognormal arriving waves and the MRC detection acts on the local Nakagami diversity multipath sub-channels. The correlation between the latter is through their gamma-distributed powers being conditioned on the corresponding arriving local average lognormal power.

In the vector form for $\mathbf{x}=\left(x_{1}, x_{2}, \ldots, x_{L}\right),(14)$ can be rewritten as

$$
M_{p, \mathrm{MRC}}(s)=\int_{0}^{\infty} \prod_{i=1}^{L} M_{\mathrm{GM}}\left(s, x_{i} / m\right) f_{\mathrm{LN}}(\mathbf{x}) d \mathbf{x} .
$$

By equating $f_{\mathrm{LN}}(\mathbf{x}) d \mathbf{x}=f_{z}(\mathbf{z}) d \mathbf{z}$ and using (13) we have equation (16) (see next page).

To de-correlate (16), we make a change of variable $\mathbf{z}=\sqrt{2} \mathbf{C}_{\mathbf{z}}^{1 / 2} \mathbf{u}+\mu_{\mathbf{z}}$ and (10) becomes (17).

where $c_{i j}$ is the $(i, j)$ element of $\mathbf{C}_{\mathbf{z}}^{1 / 2}$ which is obtained from $\mathbf{C}_{\mathbf{z}}=\mathbf{C}_{\mathbf{z}}^{1 / 2}\left(\mathbf{C}_{\mathbf{z}}^{1 / 2}\right)^{T}$ using Cholesky decomposition. Since $\mathbf{C}_{\mathbf{z}}$ is symmetric and positive definite, $\mathbf{C}_{\mathbf{z}}^{1 / 2}$ is lower triangular. 


$$
M_{p, \mathrm{MRC}}(s)=\int_{-\infty}^{\infty} \frac{1}{(2 \pi)^{L / 2}\left|\mathbf{C}_{\mathbf{z}}\right|^{1 / 2}} \prod_{i=1}^{L}\left(\frac{1}{1+\frac{s}{m} \exp \left(z_{i} / \zeta\right)}\right)^{m} \exp \left(-\frac{1}{2}\left(\mathbf{z}-\mu_{\mathbf{z}}\right)^{T} \mathbf{C}_{\mathbf{z}}^{-1}\left(\mathbf{z}-\mu_{\mathbf{z}}\right)\right)
$$

$$
M_{p, \mathrm{MRC}}(s)=\int_{-\infty}^{\infty} \frac{1}{\pi^{L / 2}} \prod_{i=1}^{L}\left[1+\frac{s}{m} \exp \left(\frac{\sqrt{2}}{\zeta} \sum_{j=1}^{L} c_{i j} u_{j}+\frac{\mu_{j}}{\zeta}\right)\right]^{-m} \exp \left(-\mathbf{u}^{T} \mathbf{u}\right) d \mathbf{u}
$$

$$
M_{p, \mathrm{MRC}}\left(s, \mu_{\mathbf{z}}, \mathbf{C}_{\mathbf{z}}\right) \approx \sum_{n_{l}=1}^{N_{p}} \ldots \sum_{n_{1}=1}^{N_{p}} \frac{w_{n_{1}} \ldots w_{n_{L}}}{\pi^{L / 2}} \prod_{i=1}^{L}\left[1+\frac{1}{m} s \exp \left(\frac{\sqrt{2}}{\zeta} \sum_{j=1}^{L} c_{i j} a_{n_{j}}+\frac{\mu_{j}}{\zeta}\right)\right]^{-m} .
$$

The integral in (17) can be accurately approximated using Gauss-Hermite expansion [14]. The MGF of the sum of $L$ correlated composite Rayleigh-lognormal power gains finally is given in (18). in which $w_{n}, a_{n}$ and $N_{p}$ are, respectively, the weights, the abscissas and the order of the Gauss-Hermite polynomial. As in [5] for the case of MRC diversity reception when only lognormal shadowing exists, in this paper we have found that (18) gives a very accurate result when $N_{p}=12$.

\subsection{Calculation of $C_{z}$ from the Lognormal Channel Covariance Matrix $\mathrm{C}_{\mathrm{LN}}$}

The same as in [5], we use the popular decreasing correlation model proposed in [15] for shadow fading channels. The covariance matrix of the channel power gains, assuming micro-diversity channels being identically distributed, is

$$
\mathbf{C}_{\mathrm{LN}}(i, j)=\operatorname{Cov}\left(p_{\mathrm{LN}_{i},} p_{\mathrm{LN}_{j}}\right)=\operatorname{Var}\left(p_{\mathrm{LN}}\right) \rho^{|i-j|},
$$

in which $\rho$ is the correlation coefficient of two adjacent channel power gains.

The following statistical relationships are well known between the lognormal random variable $p_{\mathrm{LN}}$ and its associated Gaussian random variable $Z$ :

$$
\begin{aligned}
E\left(p_{\mathrm{LN}}\right) & =\bar{p}_{\mathrm{LN}}=e^{\mu_{z} / \zeta+\sigma_{z}^{2} / 2 \zeta^{2}}, \\
\frac{\operatorname{Var}\left(p_{\mathrm{LN}}\right)}{\bar{p}_{\mathrm{LN}}^{2}} & =\left(e^{\sigma_{z}^{2} / \zeta^{2}}-1\right) .
\end{aligned}
$$

Hence

$$
\begin{aligned}
& \sigma_{z}^{2}=\zeta^{2} \ln \left(1+\frac{\operatorname{Var}\left(p_{\mathrm{LN}}\right)}{\bar{p}_{\mathrm{LN}}^{2}}\right) \\
& \mu_{z}=\zeta \ln \left(\bar{p}_{\mathrm{LN}}\right)-0.5 \zeta \ln \left(1+\frac{\operatorname{Var}\left(p_{\mathrm{LN}}\right)}{\bar{p}_{\mathrm{LN}}^{2}}\right),
\end{aligned}
$$

and

$$
\mathbf{C}_{z}(i, j)=\operatorname{Cov}\left(Z_{i}, Z_{j}\right)=\zeta^{2} \ln \left(1+\frac{\operatorname{Var}\left(p_{L n}\right) \rho^{|i-j|}}{\bar{p}_{\mathrm{LN}}^{2}}\right) .
$$

In this paper, we normalize the lognormal channel's mean power gain $\bar{p}_{\mathrm{LN}}=1$ to avoid dependency on propagation distance and adopt a fixed Gaussian standard deviation $\sigma_{z}=8 \mathrm{~dB}$. Therefore the MGF of the MRC output in (18) can be completely known in terms of the correlation coefficient $\rho$. Note that this normalization automatically gives $\bar{p}_{\mathrm{GM} \text {-LN }}=1$ since from (16), $\bar{p}_{\mathrm{GM}}=\Omega=1$ which is inherent in the composite fading model in (17) and (18).

\subsection{Estimation of Sum of Composite \\ Gamma-lognormal RVs as a Single Lognormal RV}

In this section, we approximate the sum of $L$ correlated composite gamma-lognormal RVs by a single lognormal $\mathrm{RV}, \hat{p}_{\mathrm{Ln}}=10^{0.1 \hat{Z}}$ where $\hat{Z} \sim N\left(\hat{\mu}_{z}, \hat{\sigma}_{z}^{2}\right)$. Similar approximation has been investigated for the case of independent Suzuki RVs, i.e. Nakagami with $m=1$, as a single lognormal $\mathrm{RV}$ in [2] using a two-point MGF matching technique, and as a single Suzuki RV in [16] by using the mean and variance matching technique. In a recent paper [5] we introduced a 'lossless'. two-point MGF matching technique which guarantees conservation of average signal power across the MRC combiner and which can locate the two matching points accurately, and therefore is more accurate than that proposed in [2]. By matching the MGF of the lognormal approximation $\hat{p}_{\mathrm{LN}}$ with the MGF of the sum of the composite gamma-lognormal input powers $p$ in (18) at two different positive real values $s_{1}$ and $s_{2}$, a system of two simultaneous equations as in (17) is obtained which can then be used to solve for $\hat{\mu}_{z}$ and $\hat{\sigma}_{z}^{2}$.

$$
\begin{aligned}
& \sum_{n=1}^{N_{p}} w_{n} \exp \left[-s_{i} \exp \left\{\left(a_{n} \hat{\sigma}_{z} \sqrt{2}+\hat{\mu}_{z}\right) / \zeta\right\}\right] \\
& =\sqrt{\pi} M_{p, \operatorname{MRC}}\left(s_{i}, \mu_{z}, \mathbf{C}_{\mathbf{z}}\right), \quad i=1,2 .
\end{aligned}
$$

The principle of a lossless MRC thus gives the approximated average power gain at the output of the receiver as

$$
\hat{\bar{p}}_{\mathrm{LN}}=\exp \left[\frac{\hat{\mu}_{z}}{\zeta}+\frac{1}{2}\left(\frac{\hat{\sigma}_{z}}{\zeta}\right)^{2}\right]
$$

Equation (25) provides a valid and reliable equation for iteratively improving the accuracy of the locations of the two MGF matching points $s_{1}$ and $s_{2}$. The percentage error of power loss is defined as follows [5].

$$
\text { error }(\%)=100 \frac{L-\hat{\bar{p}}_{\mathrm{LN}}}{L} \text {. }
$$




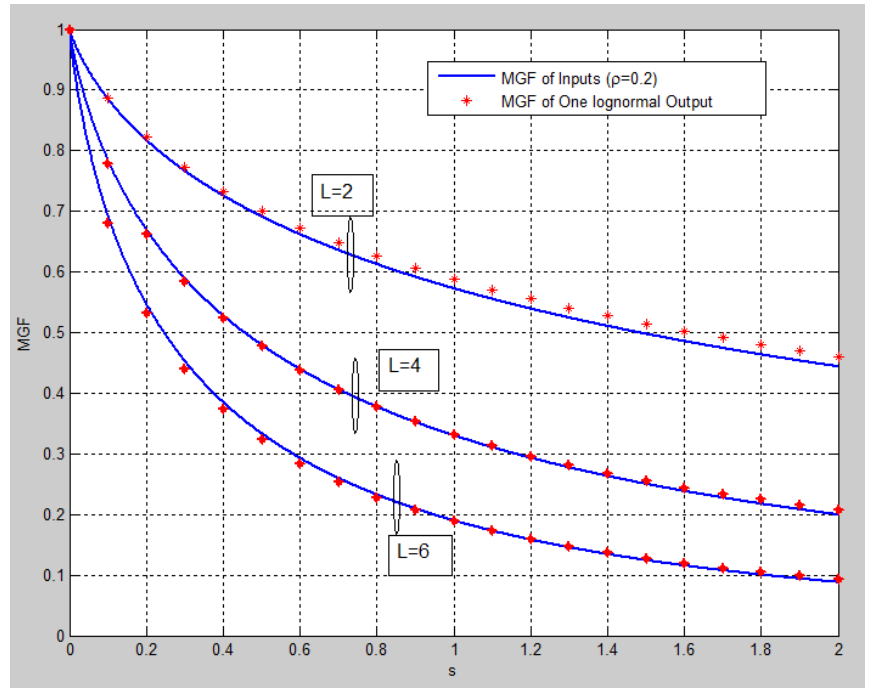

Figure 2. MGF matching of the power sum of $L$ correlated gammalognormal RVs to a single lognormal RV.

A simple iterative search algorithm for the two matching locations, hence the values of $\hat{\mu}_{z}$ and $\hat{\sigma}_{z}$, in (22) is carried out until the power loss decreases to a specified error threshold which is set at $0.1 \%$ in this paper.

The PDF of the channel power gain at the output of the MRC receiver, approximated as a single lognormal $\mathrm{RV}$, finally is

$$
\hat{f}_{\mathrm{LN}, \mathrm{MRC}}(p)=\frac{1}{p} \frac{\zeta}{\hat{\sigma} \sqrt{2 \pi}} \exp \left(-\frac{\left(10 \log _{10} p-\hat{\mu}_{z}\right)^{2}}{2 \hat{\sigma}_{z}^{2}}\right) .
$$

Table 1 presents the MGF matching results for matching points $\left(s_{1}, s_{2}\right)$ and $\left(\hat{\mu}_{z}, \hat{\sigma}^{2}\right)$ for different shadowing channel correlations $\rho$ and different multipath fading parameters $\mathrm{m}$ when the MRC output is approximated as a single lognormal RV. The error is limited to below $0.1 \%$. As discussed in the introduction, the performance parameter in this paper is the agreement in the theoretical BER and the Monte-Carlo simulated $B E R$. Therefore we want to give an equal weight, i.e. $\exp (-s p)$, as much as possible to the entire range of SNR in the MGF calculation, i.e. keeping the MGF matching points $\left(s_{1}, s_{2}\right)$ as small as possible but still satisfying the specified matching error ceiling.

Figure 2 shows the MGF matching of the corresponding solutions in Table 1 for $m=1$. It can be observed that when there are only two microdiversitybranches to add together, the sum of two exponential-distributed multipath components, i.e. a chi-square of only four degrees of freedom, is not sufficient to be approximated as a lognormal RV. As a result, the MGF matching of the sum of two gamma-lognormal RVs to a single lognormal RV cannot be expected to be as good as the matching when there are many moregamma-lognormal $\mathrm{RVs}$, i.e. a chi square of many more degrees of freedom, to sum up.

The consequence of assuming a single lognormal $\mathrm{RV}$ for the output is to skew the distribution of SNR to the lower end as demonstrated in Figure 3, giving rise to higher BER as shown in Figure 4 for $L=2$. We notice that this poor matching at low values of

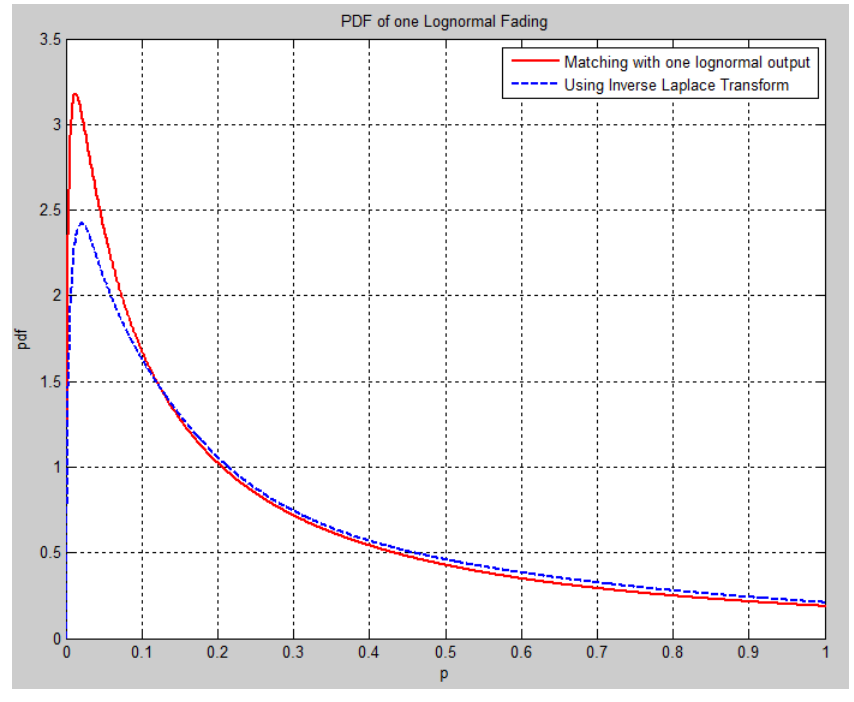

Figure 3. PDF of the single lognormal RV approximating the MRC output for $\rho=0.2$ and $N=2$.

$L$ is quite obvious but without authors'comments in Figure 7 of [3] for the sum of uncorrelated Suzuki RVs.

\subsection{BER of QPSK in Diversity Reception in Correlated Composite Nakagami-lognormal Fading Channels}

In this paper we use QPSK signaling for our analysis and simulation of mobile radio transmission using MRC diversity reception in a correlated composite Nakagami-lognormal fading environment.

Using PDF of the estimated single lognormal RV: The corresponding BER using QPSK signaling, $M=4$ and without using Gray code:

$$
\operatorname{BER}_{\mathrm{AWGN}, \mathrm{QPSK}}\left(\gamma_{b}\right)=\frac{4}{3} Q\left(\sqrt{2 \gamma_{b}}\right),
$$

where $\gamma_{b}$ is the SNR per bit and (26), is given by (27). In (27), $\gamma_{b}(p)=\frac{E_{b}}{N_{0}} p$ and term power gain $p=|h|^{2}$.

By setting

$$
u=\frac{10 \log _{10} p-\hat{\mu}_{z}}{\hat{\sigma}_{z} \sqrt{2}} \Longleftrightarrow p=\exp \left(\frac{\hat{\mu}_{z}}{\zeta}+\frac{\sqrt{2} \hat{\sigma}_{z}}{\zeta} u\right)
$$

Equation (27) can be reduced to

$$
\begin{aligned}
& \text { BER }_{\text {NG-LN,QPSK,MRC }}= \\
& \frac{1}{\sqrt{\pi}} \int_{0}^{\infty} B E R_{\text {AWGN,QPSK }}\left(\gamma_{b}(u)\right) e^{-u^{2}} d u,
\end{aligned}
$$

where $\gamma_{b}(u)=\frac{E_{b}}{N_{0}} \exp \left(\hat{\mu}_{z} / \zeta+u \hat{\sigma}_{z} \sqrt{2} / \zeta\right)$ is the argument of $\operatorname{BER}_{\mathrm{AWGN}, \mathrm{QPSK}}\left(\gamma_{b}\right)=\frac{4}{3} Q\left(\sqrt{2 \gamma_{b}}\right)$.

The above expression for BER can then be accurately approximated by an $N_{p}$-order Gauss-Hermite polynomial expansion as given below

$$
\begin{aligned}
& \text { BER }_{\text {NG-LN,QPSK,MRC }}= \\
& \frac{1}{\sqrt{\pi}} \sum_{n=1}^{N_{p}} w_{n} B E R_{\text {AWGN,QPSK }}\left(\gamma_{b}\left(a_{n}\right)\right) .
\end{aligned}
$$


Table I

Results of the 2-POINT MGF MATChing OF THE MRC OUTPUT TO A SINGLE LOGNORMAL $R$

\begin{tabular}{|l|l|l|l|l|l|}
\hline \multirow{2}{*}{$\begin{array}{l}\text { Matching } \\
\text { parameters }\end{array}$} & & \multicolumn{3}{|l|}{ Shadowing correlation $\rho=0.2$} & \multicolumn{2}{l|}{ Shadowing correlation $\rho=0.6$} \\
\cline { 2 - 6 } & & $\mathrm{m}=1.0$ & $\mathrm{~m}=2.0$ & $\mathrm{~m}=1.0$ & $\mathrm{~m}=2.0$ \\
\hline \multirow{3}{*}{$\left(s_{1}, s_{2}\right)$} & $\mathrm{N}=2$ & $(0.0065,0.0111)$ & $(0.001,1.500)$ & $(0.0178,0.0196)$ & $(0.0005,1.5000)$ \\
\cline { 2 - 6 } & $\mathrm{N}=4$ & $(0.001,0.800)$ & $(0.001,1.700)$ & $(0.025,0.800)$ & $(0.0140,2.3000)$ \\
\cline { 2 - 6 } & $\mathrm{N}=6$ & $(0.001,1.100)$ & $(0.0015,1.4500)$ & $(0.042,1.100)$ & $(0.0350,1.5000)$ \\
\hline \multirow{3}{*}{$\left(\hat{\mu}_{z}, \hat{\sigma}_{z}\right) \mathrm{dB}$} & $\mathrm{N}=2$ & $(-4.3096,0.9917)$ & $(-3.1285,0.2993)$ & $(-4.5674,8.1148)$ & $(-3.9369,0.7700)$ \\
\cline { 2 - 6 } & $\mathrm{N}=4$ & $(1.341,6.374)$ & $(2.0638,5.8660)$ & $(0.390,6.994)$ & $(0.9599,6.6376)$ \\
\cline { 2 - 6 } & $\mathrm{N}=6$ & $(4.310,5.491)$ & $(4.8126,5.0742)$ & $(3.395,6.174)$ & $(3.8155,5.8852)$ \\
\hline
\end{tabular}

$B E R_{\mathrm{NG}-\mathrm{LN}, \mathrm{QPSK}, \mathrm{MRC}}=\int_{0}^{\infty} B E R_{\mathrm{AWGN}, \mathrm{QPSK}}\left(\gamma_{b}(p)\right) \frac{1}{p} \frac{\zeta}{\hat{\sigma}_{z} \sqrt{2 \pi}} \exp \left(-\frac{\left(10 \log _{10} p-\hat{\mu}_{z}\right)^{2}}{2 \hat{\sigma}_{z}}\right)$

\section{Monte Carlo Simulation of Diversity MRC Reception in CoRrelated Composite NAKAgAmi-LOgNoRmal Fading ChanNels}

\subsection{Simulation of correlated $L$-variates lognormal vector $h_{\mathrm{LN}}$}

In this section, we simulate the correlated $L$-variates lognormal vector $h_{\mathrm{LN}}$ by first generating the $L$-variates normal vector $z=N\left(\mu_{\mathbf{Z}}, \mathbf{C}_{\mathbf{Z}}\right)$ from Section 4.2.

- Step 1: Generate i.i.d unit $L$-variates $U_{1}, U_{2}, \ldots, U_{L}$.

- Step 2: Generate correlated normal variates $Z_{1}, Z_{2}, \ldots, Z_{L}$. For each $i=1,2, \ldots, L$

$$
Z_{i}=\mu_{Z i}+\sum_{j=1}^{i} c_{i j} U_{j}
$$

where $c_{i j}$ is the $(i, j)$ element of the lower triangular $\mathbf{C}^{1 / 2}$ as in (17) obtained from matrix $\mathbf{C}_{Z}=$ $\mathbf{C}_{Z}^{1 / 2}\left(\mathbf{C}_{Z}^{1 / 2}\right)^{T}$ using Cholesky decomposition.

- Step 3: Calculate the correlated lognormal variates $X_{i}=e^{Z i}$, for $\mathrm{i}=1,2, \ldots, \mathrm{L}$. In which $X$ is the channel power gain, i.e. $\left|h_{\mathrm{LN}}\right|^{2}$.

- Step 4: Then calculate the fading transfer function of each lognormal diversity channels.

$$
h_{\mathrm{LN}}=e^{\mathrm{Zi/2}} \text {. }
$$

\subsection{Simulation of Correlated $L$-variates Nakagami- $m$ Vector $r$}

To date there is no known technique to directly generate correlated Nakagami- $m$ vector $\mathbf{r} \sim \mathbf{N G}\left(m, \mathbf{C}_{\mathbf{N G}}\right)$ except first to generate the corresponding correlated gamma vector $\mathbf{y}=\mathbf{p}_{\mathbf{G M}} \sim \mathbf{G M}\left(m, \mathbf{C}_{\mathbf{G M}}\right)$ then using the fact that a scalar Nakagami RV is equal to the square root of the corresponding gamma RV [17]. In [17], it is shown that a gamma-distributed vector has a simple direct-sum decomposition of squares of independent Gaussian vectors $\mathbf{z}_{\mathbf{k}}$, i.e

$$
\mathbf{y}=\sum_{k=1}^{2 m} \mathbf{z}_{k}^{o 2}
$$

where the notation ' $\mathrm{o}$ ' is to mean element-to-element operation, i.e. the element of the $L$-variate vector $\mathbf{y}$ is

$$
y(i)=\sum_{k=1}^{2 m} z_{k}^{2}(i), \quad i=1,2, \ldots, L .
$$

The element of the corresponding $L$-variate Nagakami- $m$ vector therefore can be generated as

$$
r(i)=\sqrt{\sum_{k=1}^{2 m} z_{k}^{2}(i)}
$$

The relationship between the covariance matrix $\mathrm{C}_{\mathrm{GM}}$ of the gamma vector and the covariance matrix $C_{z}$ of the corresponding generating Gaussian vector is [17]

$$
\mathbf{C}_{z}=\frac{1}{2 \sqrt{m}} \mathbf{C}_{\mathbf{G M}}^{o 1 / 2}
$$

From (4) with $E\left[p_{\mathrm{GM}}\right]=1$, we have $\operatorname{Var}\left[p_{\mathrm{GM}}\right]=1 / m$, and thus

$$
\mathbf{C}_{\mathbf{G M}}=\frac{1}{m}\left(\begin{array}{ccccc}
1 & \rho & \rho^{2} & \ldots & \rho^{L-1} \\
\rho & 1 & \rho^{2} & \ldots & \rho^{L-2} \\
\rho & \rho^{2} & 1 & \ldots & \rho^{L-3} \\
& \ldots & & \\
\rho^{L-1} & \ldots & \rho^{2} & \rho & 1
\end{array}\right)
$$

and

$$
\mathbf{C}_{\mathbf{Z}}=\frac{1}{2 m}\left(\begin{array}{cccccc}
1 & \sqrt{\rho} & \sqrt{\rho^{2}} & \ldots & \sqrt{\rho^{L-1}} \\
\sqrt{\rho} & 1 & \sqrt{\rho^{2}} & \ldots & \sqrt{\rho^{L-2}} \\
\sqrt{\rho} & \sqrt{\rho^{2}} & 1 & \ldots & \sqrt{\rho^{L-3}} \\
\sqrt{\rho^{L-1}} & \ldots & \ldots & \sqrt{\rho^{2}} & \sqrt{\rho} & 1
\end{array}\right) .
$$

We are now ready to generate the independent correlated Gaussian vector $\mathbf{z}_{k}, k=1,2, \ldots, 2 m$ required for (31), where

$$
z_{k}(i)=\sum_{j=1}^{i} c_{i j} U_{k}(j)
$$

where $c_{i j}$ is the $(i, j)$ element of $\mathbf{C}_{\mathbf{Z}}^{1 / 2}$ obtained from matrix $\mathbf{C}_{Z}$ in (36) using Cholesky decomposition. Note that $C_{Z}^{1 / 2}$ is a lower triangular matrix. 


\subsection{Generation of the Correlated Nakagami- $m$ Channels $h_{\mathrm{NG}}$}

The correlated $L$-variate vector $r$ in (33) only gives us the distribution of the scalar envelope of the signal in a correlated Nakagami- $m$ fading channel.

Case $m=1$ :

For the case $m=1$, the Nakagam distribution reduces to the Rayleigh distribution and the implementation of a physical Rayleigh channel $\mathbf{h}_{\mathbf{R}}$ is well understood. The gamma-distributed power gain in (33) becomes exponentially distributed $p_{R}(i)=z_{i}^{2}(i)+z_{2}^{2}(i)$ and the Rayleigh channel is implemented as

$$
h_{R}(i)=z_{i}(i)+j z_{2}(i),
$$

giving the composite (complex) Rayleigh-lognormal channel as

$$
h_{\mathrm{R}-\mathrm{LN}}(i)=h_{R}(i) h_{\mathrm{LN}}(i), \quad i=1,2, \ldots, L .
$$

Now we are ready to simulate MRC reception using QPSK signalling in a composite Rayleigh-lognormal fading environment.

Case $m=1.5,2,2.5$,

For $m>1$, the implementation of a physical Nakagami$m$ channel $h_{\mathrm{NG}}$ requires a $2 m$-dimension signal space. Therefore in the conventional 2-D space of an M-QAM constellation, we have to be contented with the scalar, i.e. envelope magnitude, channel gain given in (34),

$$
h_{\mathrm{NG}}(i)=r(i) \text {, }
$$

giving the composite (scalar) Nakagami-lognormal channel as

$$
h_{\mathrm{NG}-\mathrm{LN}}(i)=h_{\mathrm{NG}}(i) h_{\mathrm{LN}}(i), \quad i=1,2, \ldots, L .
$$

Note this means both in-phase and quad-phase components of the QPSK signal has this same channel gain.

\section{Comparison of Simulated ANd THEORETICAL BER}

In Figure 4, we compare the simulated BER result using channel model in (32) with the theoretical BER calculated from (28) for the case of correlated Rayleighlognormal diversity channels when the output of the MRC receiver is approximated by a single lognormal $\mathrm{RV}$. In both theory and simulation, we normalize the power of each channel to 1 . By comparing BER performance QPSK modulation in correlated Nakagamilognormal fading channels with $m=1,2$ and $L=$ 2, 4, 6 obtained by theoretical analysis and Monte-Calor Simulation shown in Figures 4 and 5, the precision of mathematical analysis has been shown.

\section{Conclusion}

We have successfully presented a MGF two-point matching technique used to estimate the sum of several correlated composite Nakagami-m-lognormal RVs at the output of MRC to a single lognormal. The accurate estimation has been demonstrated by choosing two

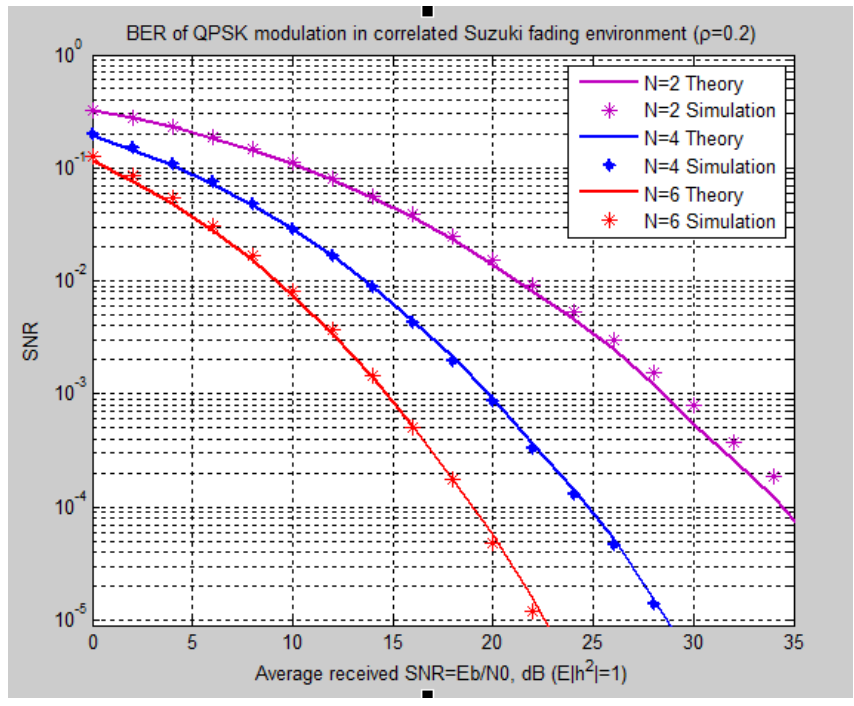

Figure 4. Comparison of theoretical BER in (28) against simulated BER for the case of correlated Suzuki-fading environment

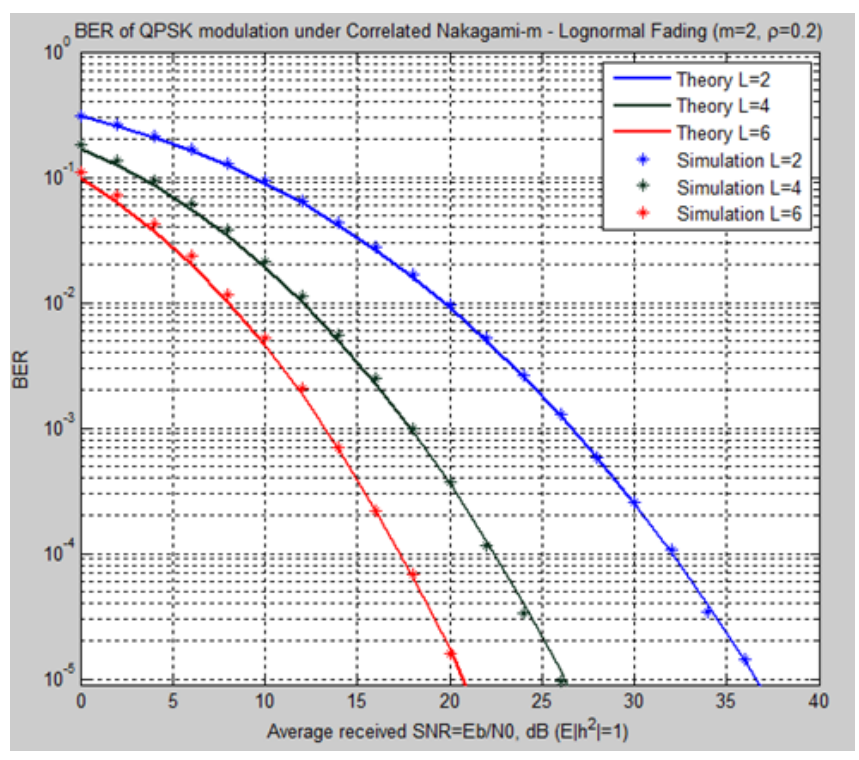

Figure 5. Comparison of theoretical BER in (28) against simulated BER for the case of correlated Rayleigh-lognormal diversity channels $(m=1$ and $m=2)$

matching points, and the value of the percentage error power loss between the average power gain at the input and output of the receiver. The higher $m$ value means the higher number of Rayleigh paths providing the better SNR. As the result, it is found that the BER is inversely proportional to the $m$. The accuracy of the mathematical analysis is also backed by Monte Carlo simulation of the BER of QPSK signal in Nakagamilognormal fading environment using MRC diversity reception.

\section{ACKNOWLEDGEMENTS}

The work is a tribute to our late supervisor, Professor Dinh Thong Nguyen, who passed away when the paper was being completed. 


\section{REFERENCES}

[1] L. Fenton, "The sum of lognormal probability distribution in scatter transmission systems," IRE Transactions on Communications Systems, vol. 8, pp. 57-67, 2003.

[2] S. Schwartz and Y. Yeh, “On the distribution function and moments of power sums with lognormal components," Bell System Technical Journal, vol. 61, pp. 1441$1462,1982$.

[3] N. B. Mehta, J. Wu, A. F. Molisch, and J. Zhang, "Approximating a sum of random variables with a lognormal," IEEE Transactions on Wireless Communications, vol. 7, pp. 2690-2699, July 2007.

[4] M. Di Renzo and F. Graziosi, "Approximating the linear combination of log-normal rvs via pearson type iv distribution for uwb performance analysis," IEEE Transactions on Wireless Communications, vol. 57, pp. 388403, February 2009.

[5] N. Quoc-Tuan, D.-T. Nguyen, and L. S. Cong, "A 10state model for an amc scheme with repetition coding in mobile wireless networks," EURASIP Journal on Wireless Communications and Networking, vol. 2013, no. 1, pp. 1-15, 2013.

[6] T. N. Quoc, T. D. Nguyen, C. L. Sinh, and M. D. T. Thai, "Two composite fading models for diversity reception in mobile wireless networks," Australian Journal of Electrical and Electronics Engineering, vol. 10, pp. 417-427, 2013.

[7] M. Nakagami, The m-distribution: A general formula of intensity distribution of rapid fading in Statistical Methods in Radio Wave Propagation. W.C. Hoffman, Ed. New York: Pergamon, 1960.

[8] M. Ho and G. Stuber, "Co-channel interference of microcellular systems on shadowed nakagami fading channels," in IEEE Vehicular Technology Conference. Secaucus, NJ, May 1993, pp. 568-571.

[9] H. Suzuki, "A statistical model for urban radio propagation," IEEE Transacsions on Communications, vol. 25, pp. 673-680, July 1997.

[10] P. X. Zhou and D. Hu, "Performance evaluations of adaptive modulation over composite nakagami-lognormal fading channels," in 15th Asia-Pacific Conference on Communications, Oct. 2009, pp. 467-470.

[11] J. Proakis, Digital Communications, 3rd ed. McGraw-Hill, 1995.

[12] C. Tellambura and A. Annamalai, "A unified numerical approach for computing the outage probability for mobile radio systems," IEEE Communications Letters, vol. 1, pp. 97-99, 1999.

[13] I. Gradshteyn and I. Ryzhik, Table of Integrals Series and Products, seventh edition ed. Academic Press, 2007.

[14] M. Abramovitz and I. Stegun, Handbook of Mathematical Functions with Formulas, Graphs, and Mathematical Tables, 9th ed. New York: Dover, 1972.

[15] M. Gudmundson, "A correlation model for shadow fading in mobile radio," IEEE Electronics Letters, vol. 27, pp. 2146-2147, 1991.

[16] J. Tighe and T. T. Ha, "On the sum of multiplicative chi square-lognormal random variables," in Proc. Globecom, vol. 6, 2001, pp. 3719-3722.

[17] Q. Zhang, "A decomposition technique for efficient generation of correlated nakagami fading channels," IEEE Journal on Selected Areas in Communications, vol. 18, pp. 2385-2392, 2000.

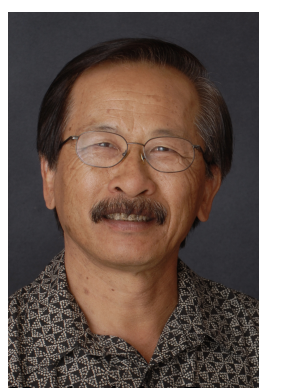

Dinh Thong Nguyen (M88) received the Bachelor of Engineering (First Class Honors) in 1965 from the University of Canterbury, Christchurch, New Zealand, and the PhD degree in antenna theory in 1969 from the University of Auckland, Auckland, New Zealand. He joined the University of Technology of South Vietnam in 1969 as an Assistant Professor. In 1975, he joined the University of Auckland, New Zealand, as an Associate Professor. In 1989, he was appointed as Professor and Head of the Department of Electrical and Electronic Engineering at the University of Tasmania, Australia, and subsequently became Executive Dean of the School of Architecture and Engineering at the same university. He retired in 2005 to Sydney, Australia, and joined University of Technology, Sydney, as an Adjunct Professor in the Faculty of Engineering and Information Technology, where he worked on a joint research project in wireless communications with the Vietnam National University, Hanoi

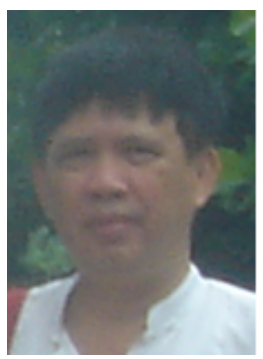

Quoc Tuan Nguyen graduated from University of Science,VNUH in 1980 and received the Master and $\mathrm{PhD}$ degrees from University of Engineering and Technology, VietNam National University HaNoi (UET, VNUH) in 1986 and 2009, respectively. He is now Professor of UET, VNUH and Head of Telecommunication Systems, Falculty of Electronics and Telecommunications, UET, VNUH.

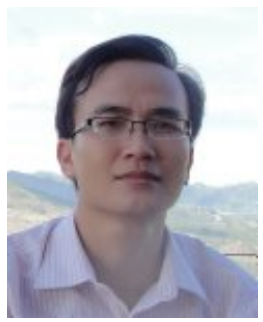

Sinh Cong Lam received the Bachelor of Electronics and Telecommunication (Honors) and Master of Electronic Engineering in 2010 and 2012,respectively from University of Engineering and Technology, VietNam National University (UET, VNUH). He is currently an $\mathrm{PhD}$ student at University of Technology, Sydney, Australia. 